\title{
The Current Status of Prostate Cancer*
}

\author{
Mukund S. Joshi \\ President, NAMS
}

\begin{abstract}
Prostate cancer (PC) is the commonest malignancy in men that causes significant morbidity and mortality. The incidence has quadrupled in the last three decades. This is predominantly due to its increased detection by excellent newer techniques like Prostate-specific antigen (PSA) evaluation, Transrectal ultrasonography (TRUS), Transrectal ultrasound-guided biopsy, Contrast enhanced ultra sound studies, Multiparametric (Mp) MRI (MpMRI) and Nuclear medicine. Its incidence shows a rise in India. With the availability of PSA and trans-rectal biopsy, nowadays the majority of prostate cancers (PC) are diagnosed at an asymptomatic early stage (T1). Most PC are adenocarcinomas while a small percentage are ductal carcinomas, mucinous carcinomas, signet ring cell carcinomas and small cell carcinomas. These variants have poor prognosis.

The anatomy of prostate will help us to further understand the basis of TRUS studies. The whole prostate can be divided into Transition Zone (TZ), Central Zone (CZ) and Peripheral Zone (PZ). This zonal anatomy of prostate is vital to understand the $\mathrm{PC}$, since $\mathrm{PC}$ is predominantly seen as follows: TZ$20 \%$; CZ-10\%; and PZ-70\%.

PSA is an extremely valuable tool in the evaluation of PC. It is exclusively produced by the prostate and to a lesser extent by the seminal vesicles. It is present in all post-pubertal men and absent in women and men following radical prostatectomy. Though the PSA is a vital parameter to detect PC, it can also be elevated in: i) Benign prostrate hypertrophy; ii) Prostate inflammation; iii) Prostatic infarct; iv) Postdigital rectal examination; and v) Sexual activity. The normal value of PSA is $0-4 \mathrm{ng} / \mathrm{mL}$. The two techniques that are available to assess PSA levels are polyclonal assay or monoclonal assay. The monoclonal assay is the most commonly used method the world over. The accepted PSA values are: $<4 \mathrm{ng} / \mathrm{mL}$ (normal); 4.0-10.00ng/mL (borderline) and $>10 \mathrm{ng} / \mathrm{mL}$ (abnormal). Other than normal PSA values, there are other PSA parameters which are often useful in confirming the diagnosis of PC. These are: i) PSA density; ii) PSA velocity; iii) PSA doubling time; iv) Other markers like PCA3; and v) $\mathrm{PC}$ is associated with more protein bound PSA(less free PSA) than in BPH.
\end{abstract}

Free PSA (FPSA) can enhance the specificity of the total PSA value for detection of the PC while reducing the number of unnecessary biopsies.

Another new finding is that of levels of insulin like growth factor binding protein-2 (IGFBP-2) appear to be directly associated with the presence of PC.

*ACADEMY ORATION delivered by Dr. Mukund S. Joshi, President, NAMS (India) during the NAMSCON 2018 held at the Mahatma Gandhi Medical College \& Research Institute, Puducherry.

Correspondence: Dr. Mukund S. Joshi, 809, Harjivandas Estate, Dr. Ambedkar Road, Dadar, Maharashtra-400014.E-mail: drmukundjoshi@gmail.com. 
Prostate Biopsy: Ultrasound guided biopsy of the prostate still remains the most important technique for the diagnosis of PC. Different biopsies which are used for diagnosis of PC are: Saturation Biopsy, MRI Guided Biopsy and Fusion Biopsy.

Use of Gleason Score for grading the PC: Gleason score is the grading system used to determine the aggressiveness of PC. This grading system can be used to choose appropriate treatment options. The tumour grades provide important information regarding how fast the cancer is likely to be growing and the likelihood of the cancer spreading to other parts of the body such as lymph nodes or bones. The pathologist assigns the grade of the tumour when he or she looks at the malignant cells under the microscope. The higher the Gleason grade, the more aggressive is the tumour.

Histopathology: Variants of usual acinar adenocarcinoma defined in 2004 by the WHO, include atrophic, pseudohyperplastic, foamy, colloid, signet ring, etc. Recently, variants not included in the 2004 WHO classification are microcysticadeno carcinoma, prostatic intraepithelial neoplasia adenocarcinoma, large cell neuro endocrine carcinoma and pleomorphic giant cell carcinoma.

Other diagnostic modalities for PC are Colour Flow Imaging, Elastography, Contrast Enhanced Ultrasound (CEUS), MR Imaging of Prostate Malignancies. The MR Imaging consisting of the following:

Prostate Imaging Reporting and Data System (PIRADS) refers to a structured reporting scheme for evaluating the prostate for PC.

T1-weighted images are not helpful in differentiating different zones or detecting the lesion. However, invasion of neurovascular bundle, haemorrhage within the gland and loco-regional lymphadenopathy is better visualized on this sequence.

DWI imaging plays an important role in determining PIRADS score, predominantly in peripheral zone neoplasm. It utilizes proton diffusion properties in water to produce image contrast. Thus prostate malignancy appears bright (hyperintense) on DWI with corresponding low values on ADC map (darkhypo intense). Of all functional MR imaging techniques, DW imaging is the most practical and simple in its use.

Dynamic Contrast Scan is considered positive if a suspected lesion/ nodule on T2-W or DWI image reveals earlier than normal or more than normal enhancement (hyper enhancement), as routinely seen in lesions with malignant etiology.

MR Spectroscopy reflects resonance frequencies that are unique for protons in different metabolites present at the sampled location. A change in the ratios of concentrations of these metabolites suggests abnormality within the tissue. Normal PZ has high concentration of citrate and polyamines and low concentration of choline and creatinine. A reversal of these, i.e. decrease in citrate peak due to altered metabolism and increase in choline and creatinine peaks in a suspected nodule on T2-W image may suggest malignancy. Recent studies reveal that MR spectroscopy is more specific and less sensitive that anatomic T2-W scan.

\section{Current Modalities of Prostate Cancer Treatments}

There are a wide variety of treatments available for the management of prostate cancer. Radical prostatectomy, external beam radiation and radioactive prostate seed implant are potential cures for the prostate cancer. Hormone therapy may force the cancer into a prolonged remission but does not provide a cure unless it is combined with other treatments. The most commonly used treatments include the 
following:

- Watchful Waiting (Active surveillance)

- Radical Prostatectomy (Robot assisted radical prostatectomy; Laparoscopic prostatectomy)

- Radiation Therapy (External beam radiation; Radioactive prostate seed implants)

- Hormone Therapy

- Combination of Therapies

- High Intensity Focused Ultrasound (HIFU) or Magnetic Resonance-guided Focussed Ultrasound Surgery (MRgFUS)

- Others (Cryotherapy; Photodynamic therapy)

- Metastatic Disease

1. Hormonal therapy (orchidectomy)

2. Anti androgens

3. Luteinizing Hormone-releasing Hormone (LHRH) Agonists (Leuprolide; Goserelin; Triptorelin; Histrelin) - monthly to annual depot injection S.C. implants

Metastatic PC responds to androgen- ablation/deprivation therapy, which heralded the beginning of a new era PC therapy.

Keywords: Prostate cancer, transrectal ultrasonography, transrectal ultrasound-guided prostate biopsy, prostate-specific antigen (PSA), anti-androgen therapy of prostate cancer.

\section{Introduction}

Prostate cancer is the commonest malignancy in men that causes significant morbidity and mortality. The incidence has quadrupled in the last three decades. This is predominantly due to excellent newer techniques like Prostate-specific antigen (PSA) evaluation, Transrectal ultrasonogrphy (TRUS), Transrectal biopsy, contrast enhanced ultra sound studies, MpMRI and nuclear medicine. Its incidence shows arise in India. It is the second leading site of cancer in Indian cities like Delhi, Kolkata and Pune. It is the $3^{\text {rd }}$ leading site in cities like Mumbai and Bangalore. In India, it has an incidence rate of 3.9 per $1,00,000$ men and is responsible for $9 \%$ of cancer related mortality. Prostate imaging and interpretation is based on prostate imaging, reporting and data system. With the availability of PSA and trans-rectal biopsy, nowadays the majority of prostate cancers are diagnosed at an asymptomatic early stage (T1). Most cancers of the prostate are adenocarcinomas while a small percentage are ductal carcinomas, mucinous carcinomas, signet ring cell carcinomas and small cell carcinomas.
These variants have poor prognosis.

It is well said that the discovery which will cause the greatest impact on our field would be the development of accurate imaging of a tumour within the prostate. New biological markers such as PSA 3 are thought to be promising for positive biopsies that will once again cut down the unindicatednumber of biopsies.

\section{"PROSTATE CANCER IS CONSIDERED AS THE BIGGEST ENEMY OF A GENTLEMAN."}

\section{Anatomy of Prostate}

The anatomy of prostate and further discussions are based on transrectal ultrasound studies. A quick anatomical detail was thought necessary before I proceed further.

\section{Transition Zone (TZ)}

The transition zone in the earlier years of life contains approximately $5 \%$ of prostatic 
glandular tissue. In the ageing prostate, the TZ shows marked hyperplasia and constitutes the majority of the overall prostatic glandular elements. It is located on both sides of the urethra. Prostatic calcification within the periurethral glands in the proximal urethra and verumontanum produce the typical 'Eiffel tower' effect. About $20 \%$ of prostatic cancers arise within the transition zone. These are embedded in the longitudinal smooth muscle of the proximal urethra, which is also known as the internal prostatic sphincter.

\section{CentralZone (CZ)}

The central zone is pyramidal in shape and consists of approximately $25 \%$ of glandular tissue. It is located near the base of the prostate. Its apex is at the verumontanum. Ducts from the $\mathrm{CZ}$ radiate from the base of the gland and terminate in the proximal urethra. The ejaculatory ducts pass through the $\mathrm{CZ}$ and terminate into the urethra at the verumontanum. The CZ is resistant to disease process and is the site of origin of $10 \%$ of all prostatic cancers.

\section{Peripheral Zone (PZ)}

The peripheral zone is the largest of the glandular zones, contains $70 \%$ of prostatic glandular tissue and surrounds the distal urethra. It occupies the prosterolateral and apical regions of the prostate and extends anteriorly. The ducts of the PZ enter the distal urethra, distal to the verumontanum. The $\mathrm{PZ}$ is homogeneous in echotexture. About $70 \%$ of prostatic cancers originate in the PZ. The majority of these are located in close proximity of the prostatic capsule. They are separated from the central and transition zones by the surgical capsule.

\section{Anterior Fibfomuscular Stroma (AFMS)}

The AFMS is non-glandular tissue, which forms the anterior surface of the prostate. It is situated anterior to the urethra and is composed of smooth muscle that is continuous with the detrusor muscle fibers. It is thickest just distal to the verumontanum where it mainly consists of fibrous tissue. It becomes thinner as it reaches the apex of the prostate.

Over the years, TRUS has been very useful along with PSA findings in predicting prostate volume and detecting prostate cancer lesions. Currently, MRI finds its clinical applications in all aspects in prostate cancer evaluation(1).

This zonal anatomy is vital to understand since cancer is predominantly seen as follows:
1. $\mathrm{TZ}-20 \%$
2. $\mathrm{CZ}-10 \%$
3. $\mathrm{PZ}-70 \%$

\section{Prostate-specific Antigen}

PSA is an extremely valuable tool in the evaluation of prostate cancers. It is exclusively produced by the prostate and to a lesser extent by the seminal vesicles. It is present in all post pubertal men and absent in women and men following radical prostatectomy.

PSA is a glycoprotein of low molecular weight $(35,000 \mathrm{D})$ produced by the acinii and ducts of the prostate grand. It is the tumour marker currently used for early detection of prostate cancer. Disruption of normal prostatic architecture results in elevated serum PSA levels which otherwise is rather low. Prior to the availability of PSA, the detection of prostatic cancer was mainly based on DRE and serum acetate phosphate. With the availability of PSA testing, 32 to $35 \%$ of patients with clinically significant prostatic cancer can be diagnosed beforethe disease is palpable. In more than $90 \%$ of patients, prostate cancer is diagnosed before symptoms occur.

Though the PSA is a vital parameter to detect prostate cancer, it can also be elevated in

1. Benign prostrate hypertrophy

2. Prostate inflammation 
3. Prostatic infarct

4. Post digital rectal examination

5. Sexual activity

The normal value of PSA is $0-4 \mathrm{ng} / \mathrm{cc}$. The two techniques that are available to assess PSA levels are polyclonal assay or monoclonal assay. The monoclonal assay is the most commonly used method the world over. The accepted PSA values are:

$\begin{array}{cl}<4 \mathrm{ng} / \mathrm{mL} & \text { - normal } \\ 4.0-10.00 \mathrm{ng} / \mathrm{mL} & \text { - borderline } \\ >10 \mathrm{ng} / \mathrm{mL} & \text { - abnormal }\end{array}$

The importance of PSA values is vital in deciding the line of treatment for a given cancer. It also guides as to whether to perform a prostate biopsy or otherwise. The diagnosis of prostate biopsy is not specific or absolute with PSA studies. Normal PSA does not necessarily mean that the patient has no cancer.

Similarly, abnormal values are not definitive for the presence of prostate malignancy. Of the several new entities which are useful in the diagnosis of prostate cancer in a doubtful patient, free to total PSA ratio is being advocated and practiced often by several urologists. Normally PSA in the blood is partly free and partly protein bound, thus the ratio of $3: 4$ tends to be high in benign lesions while it is low in cancer. The necessity of using this parameter is to reduce the number of biopsies and to detect cancer with higher degree of confidence. No definitive cut off value has yet been accepted. In nutshell the accepted predicted values in different ages are as follows:

\begin{tabular}{|c|c|}
\hline Age (years) & $\begin{array}{c}\text { Normal range of } \\
\text { PSA }(\mathrm{ng} / \mathrm{mL})\end{array}$ \\
\hline $42-49$ & $0.0-2.5$ \\
\hline $50-59$ & $0.0-3.5$ \\
\hline $60-69$ & $0.0-4.5$ \\
\hline $70-79$ & $0.0-6.5$ \\
\hline
\end{tabular}

Other than normal PSA values, there are other PSA parameters which are often useful in confirming the diagnosis of prostate cancer. These are

- PSAdensity

- PSA velocity

- PSA doubling time

- Other markers like PSA 3

- Prostate cancer is associated with more protein bound PSA (less free PSA) than in $\mathrm{BPH}$.

i) $\mathrm{F} / \mathrm{T}$ ratio is lower in patients with prostate cancer. This is of great value since it can improve the results of the test specificity. It is also of great value when total PSA is in the range of 4-10 $\mathrm{Ng} / \mathrm{CC}$.

In nutshell the importance of PSA as a diagnostic tool, which maybe summarised as:

(a) Screening

(b) Staging

(c) Prognostic indicator

(d) Surveillance in prostatic cancer.

The importance of PSA especially in patients who are asymptomatic are as follows:

\begin{tabular}{|c|c|}
\hline PSA & Probability $\%$ \\
\hline 0.5 & 6.60 \\
\hline $6-1$ & 10 \\
\hline $1.1-2$ & 17 \\
\hline $2.1-3$ & 24 \\
\hline $3.1-4$ & 27 \\
\hline $4-10$ & $25-30$ \\
\hline$<10$ & $42-64$ \\
\hline$>20$ & 87 \\
\hline
\end{tabular}

Free (FPSA) can enhance the specificity of the total PSA value for detection of the prostate cancer while reducing the number of unnecessary biopsies

Another new finding is that of levels of insulin like growth factor binding protein-2 (IGFBP-2) appear to be directly associated with the presence of prostate cancer (2). 


\section{Prostate Biopsy}

Ultrasound guided biopsy of the prostate still remains the most important technique for the diagnosis of prostate cancer. A rising PSA or an elevated PSA remains to date the most vital technique to diagnose prostate cancer. The previously carried out sextant biopsy has now increased to a minimum of 12 core biopsy technique. This has improved the sensitivity of prostate cancer detection. Predominantly the samples are taken from the peripheral zone. Most workers do not take TZ biopsies as a routine. A TZ biopsy is undertaken in patients where the initial systematic sampling has been negative. The routine transition biopsy has a low yield of cancer ranging from $0.6-1.0 \%$. The indications for trans-rectal biopsy of the prostate may be summarised (3) as:
a. Abnormal DRE
b. elevated or rising total PSA
c. Previous negative biopsy but high clinical suspicion for prostate cancer
d. Focal capsular bulge

\section{Prostate Evasive Anterior Tumours}

A significant number of anteriorly located cancers are diagnosed relatively late. These are missed on trans-rectal biopsy, as the biopsies tend to be laterally directed focusing mainly on the PZ. These hidden cancers are located anterior to the urethra in the TZ (49\%), anterior horns of PZ $(36 \%)$ or both $8 \%)$. These tumours are suspected when high or increasing PSA levels are present despite repeatedly negative biopsies. These patients are often kept under close surveillance. MRI is now recommended to locate such cancers after adequate anterior and TZ biopsies have failed.

\section{Saturation Biopsies}

Considering the difficulties in routine biopsies, there are many urologists who suggest and practice Saturation biopsies (24 to 40 cores). However, this technique has not been well accepted, fallen out of repute and most urologists have come back to $10 / 12$ core biopsies.

With the assistance of elastography, Doppler and contrast enhanced imaging, there is significant increase in the correct diagnosis of prostate cancers. As a result of the use of these modalities, the number of biopsies have also been reduced.

\section{MRI Guided Biopsy}

MRI guided biopsy has a constant high cancer detection rate independent of the number of previous negative TRUS biopsies as well as the number of removed prostatic cores. Assuming that MRI guided biopsy provides an accurate diagnosis in a high proportion of patients while being less invasive than repeated TRUS guided biopsies, this technique is an attractive alternative diagnostic tool for the selected group of patients who have at least one negative prior TRUS guided biopsy with persistent suspicion of prostate cancer.

\section{Fusion Biopsy}

MRI has a moderately high sensitivity and specificity for the detection of prostate cancer. Recently, a new technique has emerged which allows a pre-performed MRI to coregister to landmarks so that real time virtual ultrasound guided biopsies can be performed. Experience is limited but this is a very promising development that would overcome the limitations of TRUS in detecting cancer while retaining the flexibility and convenience of TRUS directed needle biopsy or ablative therapy $(4,5)$.

The technique allows multiplanar biopsy planning and Xu et al showed an accuracy of 2.4$1.2 \mathrm{~mm}$ in phantom and canine studies with a rate of $95.8 \%$ with a significantly higher positivity rate than non-targeted cores. Further multicentre trials are necessary to evaluate this technique in cancer detection. 


\section{Gleason Score}

Gleason score is the grading system used to determine the aggressiveness of prostate cancer. This grading system can be used to choose appropriate treatment options.

The most commonly used grading system has two grades. Each patient's tumour is assigned two grades that represent the major and minor patterns of malignant glands seen under the microscope. Each of the two grades will range from 1-5, the two grades are then added together to give a Gleason score (which ranges from 2-10). A Gleason score of 2-5 represents a low grade malignancy. These tumours are usually slow growing and have a low likelihood of spreading and are rarely fatal. Tumours that have a Gleason score of 8-10 are high grade and are more likely to be fast growing and metastasize to lymph nodes or bones. Most patients have intermediate grade tumours or a Gleason score of 6 or 7 . These are medium growing tumours and the prognosis is generally very good. The Gleason score strongly influences which staging test should be ordered and what treatment options should be considered.

The tumour grades provide important information regarding how fast the cancer is likely to be growing and the likelihood of the cancer spreading to other parts of the body such as lymph nodes or bones. The pathologist assigns the grade of the tumour when he or she looks at the malignant cells under the microscope. The higher the Gleason grade, the more aggressive is the tumour.

\section{Importance of Gleason Score}

- Useful for predicting the behaviour of a prostate cancer

- PSAlevel

- Findings from rectal examination

- The number of biopsies core samples

- Is cancer found in one or both sides?

- Has the cancer spread outside the gland?

\section{Prognostic Grade Grouping}

- Gleason Score 2 to 6 - Prognostic grade Group1/V

- Gleason Score 3+4=7-Prognostic grade Group II/V

- Gleason Score 4+3=7-Prognostic grade Grade III/V

- Gleason Score 8, Prognostic grade Grade IV/V

- Gleason Score 9-10- Prognostic grade Grade V/V

\section{Histopathology}

Variants of usual acinar adenocarcinoma defined in 2004 by the WHO, include atrophic, pseudohyperplastic, foamy, colloid, signet ring etc. Recently, variants not included in the 2004 WHO classification are microcysticadeno carcinoma, prostatic intraepithelial neoplasia adeno carcinoma, large cell neuro endocrine carcinoma and pleomorphic giant cell carcinoma (6).

\section{Colour Flow Imaging}

The normal prostate gland has little but usually bilaterally symmetric flow. The neurovascular bundles are also well identified at theinfero-lateral margins of the prostate. The neurovascular bundles, pericapsularand periurethral arteries show strong colour signals. In the initial stages, it was thought that colour flow signals especially with new machines with excellent software would solve the problem of detecting with certainty the problem of prostate cancer. However colour Doppler studies have no definite role to play in the diagnosis of prostate cancer. It has been realised that it has poor specificity with some malignancies being hypovascular while some benign lesions show increased vascularity. The commonly seen patterns in cancer of prostate colour-flow imaging are

1. Increased focal vascularity

2. Diffuse increased vascularity 
3. Increased vascularity in the tissue surrounding the lesion.

Colour-flow imaging illustrates Macrovascularity (Perfusion) thus its usefulness are

- Localisation of cancer

- Capsule penetration

- Locally advanced diseases

- Differentiates between fibrotic tissue and local recurrence.

\section{Elastography}

This is an important new technique which depends on the stiffness of a given tissue. This really amounts to palpation of a given mass (Soft, Firm and Hard). The tumour can be imaged and quantified by measuring its strain under applied pressure (compression by transducer). Cancerous tissue reveals relatively increased stiffness as a result of increased cell density. In strain imaging, the images are obtained with and without manual compression of prostate. The degree of displacement (strain produced as a result) is used to generate an elastogram (color maps) in real time. Since a tumour is usually harder as compared to the surrounding tissue, it presents as a hypoechoic (black) area. This has been very useful especially in considering trans-rectal biopsies. The accuracy in detection of prostate cancer is about $80-85 \%$.

In shear wave elastography technique, the shear waves are produced which travel at a right angle to the insonating beam. These travel faster in the stiffer tissues and thus provide a measure of tissue elasticity in quantitative terms. This technology is considered superior and more reproducible than strain imaging it has a high sensitivity and specificity. Shear wave elastography may also have better potential as this technique allows true quantification rather than strain, a surrogate index of stiffness. Shear wave elasticity helps in reducing the number of biopsies.

To summarise, Elastography is useful in
- Detection of prostate cancer

- Useful for targeted biopsy

- Staging of prostate cancer (presence or absence of extra capsular disease) (7)

\section{Contrast Enhanced Ultrasound}

Angiogenesis is known to be essential for tumour growth and invasion, in a pathology specimen. Prostate tumours show increased microvascular density as compared with the normal parenchyma. Thus, an imaging investigation that allows quantification of blood flow in these micro vessels provides an opportunity to significantly increase the detection and characterisation of a prostatic cancer (8).

This is quite essential especially since the technological advances have shown significant false negative rates today. As a result it has been observed that newer biopsy techniques and technological advances have initiated further newer techniques to detect prostate cancer at high rates. With experience, it has been observed that with newer biopsy techniques, a significant number of false negative rates have regressed (9).

With the use of ultrasound contrast agents the number of unnecessary biopsies has decreased and also their morbidity.

Ultrasound contrast techniques allow imaging of vessels down to 50 to 100 micromilimetersin diameter. One of the greatest advantages is that these contrast agents are always intra-vascular and do not extravasate beyond the lumen of blood vessels. Thus there are hardly any side effects. Of the many indices that have been studied, the time-peak enhancement is the most predictive parameter for the localisation of the malignant lesion in the prostate which is $80 \%$ correctly diagnosed. Recently targeted microbubbles are under development and will certainly improve the sensitivity of certain specific markers. An important use of these contrastsis to utilise the 
microbubbles for targeted biopsies. Though this has improved the sensitivity of detection of prostate cancer, it still does not avoid systemic biopsies. In short, further improvements are needed in increasing the sensitivity and specificity of these contrast agents.

Contrast agents are microbubbles of gas in an encapsulated cell. These are blood pool agents which remain confined to the vascular lumen until they disintegrate.

Tumour growth induces neovascularity and increased microvascular density, thus induces altered perfusion patterns that improve cancer detection. Practically, the increased density (rapid uptake) is followed by a quick wash out - CLASSICAL OF CANCER. As mentioned above, cancers have increased microvascularity. These agents enhance the area of cancer outlining the lesion in comparison to the surrounding normal tissue. Contrast enhanced targeted biopsy is one of the greatest advantages of the contrast agents.

In conclusion, the new contrast specific ultrasound techniques show promising results. One of the downsideof CEUS is the subjective interpretation by the investigators. The most important pattern which correlates with the presence of prostate cancer takes place within seconds of injection. This often makes the diagnosis of prostate cancer difficult. Much as a large group of workers believe that there is a significant increase in cancer detection rate using CEUS targeted biopsies compared to random biopsies, many scientists have reservation of the value of CEUS in this observation (10).

Microbubbles will soon be studied for quantification in prostate cancer.Similarly, they can also be used to transport certain substances. Ultrasound assisted drug delivery is being studied with great interest and will be an important usage of this very useful newer technology (11).

\section{MR Imaging in Prostate Malignancies}

Prostate cancer is the most common malignancy in men. In recent years, prostate cancer is commonly detected early, hence imaging plays an important role in locating and delineating the extent of the disease. MRI is very useful in evaluating primary tumour, metastasis and recurrence. MRI allows detection of extracapsular spread of the disease, seminal vesicle invasion and simultaneous screening of regional lymph nodes and pelvic bones (12).

A multiparametric MR imaging prostate examination consists of T1- and T2-weighted imaging combined with one or more functional MR imaging techniques (dynamic contrast agent-enhanced MR imaging, diffusionweighted (DW) imaging, and hydrogen 1 MR spectroscopic imaging) (13).

T2-weighted MR imaging is the workhorse of prostate MR imaging. T2weighted images have high spatial resolution and, thus, can clearly differentiate the normal intermediate to high signal intensity peripheral zone from the low-signal-intensity central and transition zones in young male subjects. A neoplastic lesion is hypo intense on T2-W scan.

T1-weighted images are not helpful in differentiating different zones or detecting the lesion. However invasion of neurovascular bundle, haemorrhage within the gland and loco regional lymphadenopathy is better visualized on this sequence.

DWI imaging plays an important role in determining PIRADS score, predominantly in peripheral zone neoplasm. It utilizes proton diffusion properties in water to produce image contrast. Thus prostate malignancy appears bright (hyper intense) on DWI with corresponding low values on ADC map (darkhypo intense). Of all functional MR imaging techniques, DW imaging is the most practical and simple in its use. 
Dynamic contrast scan is considered positive if a suspected lesion/ nodule onT2-W or DWI image reveals earlier than normal or more than normal enhancement (hyper enhancement), as routinely seen in lesions with malignant etiology.

MR spectroscopy reflects resonance frequencies that are unique for protons in different metabolites present at the sampled location. A change in the ratios of concentrations of these metabolites suggests abnormality within the tissue. Normal peripheral zone has high concentration of citrate and polyamines and low concentration of choline and creatinine. A reversal of these i.e. decrease in citrate peak due to altered metabolism and increase in choline and creatininepeaks in a suspected nodule on T2W image may suggest malignancy. Recent studies reveal that MR spectroscopy is more specific and less sensitive that anatomic T2-W scan.
PI-RADS (Prostate Imaging Reporting and Data System) refers to a structured reporting scheme for evaluating the prostate for prostate cancer (14). Categories of its scoring are given below (Table 1,2,3):

- PI-RADS 1: very low (clinically significant cancer is highly unlikely to be present)

- PI-RADS 2: low (clinically significant cancer is unlikely to be present)

- PI-RADS 3: intermediate (the presence of clinically significant cancer is equivocal)

- PI-RADS 4: high (clinically significant cancer is likely to be present)

- PI-RADS 5: very high (clinically significant cancer is highly likely to be present)

The PI-RADS score for peripheral gland lesion is predominantly acquired by DWI scan and for the central gland lesion is predominantly acquired by $\mathrm{T} 2 \mathrm{~W}$ scan.

PI-RADS scoring system is a standard subset of MR scan of prostate to grade the risk of malignancy.

Table 1: Details of the PI-RADS scoring system

\begin{tabular}{|c|l|l|}
\hline Score & Peripheral Zone (PZ) & Transition Zone (TZ) \\
\hline 1 & $\begin{array}{l}\text { Uniform hyper intense signal } \\
\text { intensity ( Normal ) }\end{array}$ & $\begin{array}{l}\text { Homogeneous intermediate signal intensity } \\
\text { (Normal) }\end{array}$ \\
\hline 2 & $\begin{array}{l}\text { Linear or wedge shaped hypo } \\
\text { intensity or diffuse mild hypo } \\
\text { intensity, usually indistinct } \\
\text { margin }\end{array}$ & $\begin{array}{l}\text { Circumscribed hypo intense or } \\
\text { heterogeneous encapsulated nodule(s) (BPH) }\end{array}$ \\
\hline 3 & $\begin{array}{l}\text { Heterogeneous signal intensity or } \\
\text { non-circumscribed, rounded, } \\
\text { moderate hypo intensity }\end{array}$ & $\begin{array}{l}\text { Heterogeneous signal intensity with } \\
\text { obscured margins }\end{array}$ \\
\hline 4 & $\begin{array}{l}\text { Circumscribed, homogeneous } \\
\text { focus/mass confined to prostate } \\
\text { and }<1.5 \mathrm{~cm} \text { in greatest } \\
\text { dimension }\end{array}$ & $\begin{array}{l}\text { Lenticular or non -circumscribed, } \\
\text { homogeneous, moderately hypo intense, and } \\
<1.5 \text { cm in greatest dimension }\end{array}$ \\
\hline 5 & $\begin{array}{l}\text { Same as 4, but } \geq 1.5 \mathrm{~cm} \text { in } \\
\text { greatest dimension or definite } \\
\text { extraprostatic extension/invasive } \\
\text { behaviour }\end{array}$ & $\begin{array}{l}\text { Same as 4, but } \geq 1.5 \text { cm in greatest } \\
\text { dimension or definite extraprostatic } \\
\text { extension/invasive behaviour }\end{array}$ \\
\hline
\end{tabular}


Table 2: PI-RADS scoring for peripheral gland lesions

\begin{tabular}{|l|l|l|l|}
\hline DWI & T2W & DCE & PI-RADS score \\
\hline 1 & Any & Any & 1 \\
\hline 2 & Any & Any & 2 \\
\hline 3 & Any & - & 3 \\
\cline { 2 - 4 } & & + & 4 \\
\hline 4 & Any & Any & 4 \\
\hline 5 & Any & Any & 5 \\
\hline
\end{tabular}

Table 3: PI-RADS scoring for central gland lesions

\begin{tabular}{|l|l|l|l|}
\hline T2W & DWI & DCE & PI-RADS score \\
\hline 1 & Any & Any & 1 \\
\hline 2 & Any & Any & 2 \\
\hline 3 & $\leq 4$ & Any & 3 \\
\cline { 2 - 4 } & 5 & Any & 4 \\
\hline 4 & Any & Any & 4 \\
\hline 5 & Any & Any & 5 \\
\hline
\end{tabular}

Table 4: Details of PI-RADS v2 scoring system

\begin{tabular}{|c|l|}
\hline Score & Peripheral Zone (PZ) OR Transition Zone (TZ) \\
\hline 1 & No abnormality on ADC and high b-value DWI \\
\hline 2 & Indistinct hypo intense on ADC \\
\hline 3 & $\begin{array}{l}\text { Focal mild-moderate hypo intense on ADC and iso-mildly hyper intense on } \\
\text { high b-value DWI }\end{array}$ \\
\hline 4 & $\begin{array}{l}\text { Focal markedly hypo intense on ADC and markedly hyper intense on high } \\
\text { b-value DWI; }<1.5 \text { cm in greatest dimension }\end{array}$ \\
\hline 5 & $\begin{array}{l}\text { Same as } 4 \text { but }>1.5 \text { cm in greatest dimension or definite extraprostatic } \\
\text { extension }\end{array}$ \\
\hline
\end{tabular}

The original PI-RADS score was annotated, revised and published as the second version, PI-RADS v2, by a steering committee comprising the joint efforts of the American College of Radiology (ACR), European Society of Urogenital Radiology (ESUR), and
AdMeTech Foundation (Table 4).

MR guided biopsy is a promising tool in patients with one or more previous negative systematic random biopsy sessions. Transrectal MR-guided biopsy improves prostate cancer 
detection; however, its availability is limited, and examination times are long. MR guidance of prostate biopsy may improve determination of the true pre-treatment Gleason score (15).

In conclusion, although reported accuracies of different components of multiparametric MR imaging techniques are inconsistent, in general the addition of multiparametric MR imaging techniques to T2weighted MR imaging improves accuracy for both localization and local staging of prostate cancer.

Of all clinical indications for multiparametric MR imaging of the prostate, localization is the most important. Accurate localization of prostate cancer results in more accurate prostate cancer staging and MR guidance of prostate biopsy and therapy. MRI is also very helpful in determining recurrence of malignancy and restaging of prostate cancer. PIRADS v2 is the current standard method for evaluating pre-treatment prostate lesions and determining the risk of malignancy.

\section{Role of Prostate Specific Membrane Antigen (PSMA)}

PSMA is a transmembrane glycoprotein with enzymatic properties which is expressed hundred to thousand-fold in prostate cancer. It is present very weakly in normal prostatic tissue and gets even more expressed in androgen resistant prostate cancer.

It can be imaged by Nuclear medicine techniques using 68Ga-PSMA or 18F-PSMA which are PET tracers. It has high sensitivity and specificity for imaging prostate cancer.

Over $90 \%$ of prostate cancers express PSMA. Expression may be low in low grade cancers or cancers which have undergone neuroendocrine differentiation.

PSMA PET-CT scan is becoming the diagnostic test of choice as a "one stop shop" in the prostate cancer imaging (16). It has a role in:

1. Initial diagnosis of prostate cancer

2. Detection of recurrence after prostatectomy or radiation therapy in a patient with rising PSA(biochemical recurrence)

3. Follow-up response to chemotherapy or radiation therapy

In the initial diagnosis of prostate cancer in a symptomatic or asymptomatic patient with elevated PSA, PSMA PET-CT performs as well as a multiparametric MRI (MpMRI) which currently is considered the test of choice. There is a 97\% concordance between PSMA PET-CT and MpMRI in this setting. The advantage of MpMRI is its ability to show involvement of the neurovascular bundle, which is difficult to detect on PSMA PET-CT due to inferior spatial resolution. However, PSMA PET-CT outperforms MRI and CT in N and M staging.

Over $80 \%$ of lymph nodal metastases in prostate cancer occur in nodes less than $8 \mathrm{~mm}$. $\mathrm{CT}$ and MRI depend on size criteria for diagnosing metastases, hence would not detect all the lymph nodal metastases. PSMA PET-CT can detect nodal involvement in as small as $3 \mathrm{~mm}$ node (17).

In the setting of biochemical recurrence after radical prostatectomy and radiation therapy, PSMA PET-CT has great sensitivity of $60 \%$ for detection of recurrence site compared to almost zero with other modalities. At PSA levels $<2 \mathrm{ng} / \mathrm{mL}$, the detection sensitivity is greater than 97\%. PSMA PET-CT also identifies patients, who would be suitable for 177 lutium PSMA therapy for refractory metastatic prostate cancer.

\section{An Overview of Prostate Cancer Treatments}

There are a wide variety of treatments available for the management of prostate cancer. Radical prostatectomy, external beam radiation and radioactive prostate seed implant are 
potential cures for the prostate cancer. Hormone therapy may force the cancer into a prolonged remission but does not provide a cure unless it is combined with other treatments. The most commonly used treatments include the following:

- Radical prostatectomy

- Robot assisted radical prostatectomy

- Laparoscopic prostatectomy

- External beam radiation

- Radioactive prostrate seed implants

- Hormone therapy

- Watchful waiting

- Combination of therapies

- High intensity focussed ultrasound (HIFU) -guided thermal ablation

- Others-cryotherapy, photodynamic therapy

- Metastatic disease

1. Hormonal therapy (orchidectomy)

2. Anti androgens

3. LHR analogs (ZOLODEX, LUPRON)

Metastatic Prostate cancer responds to androgen ablation therapy, which heralded the beginning of a new era prostate cancer therapy (18).

Medical castration with oral androgens became the first effective systematic treatment for any cancer and to date the androgen ablation remains the most generally useful prostate cancer therapy.

\section{High Intensity Focussed Ultrasound (HIFU) Guided Thermal Ablation \\ or}

Magnetic Resonance Guided Focussed Ultrasound Surgery (MRgFUS) for CA Prostate

\section{Principle and Rationale}

Traditionally accepted methods for treatment of Carcinoma prostate have been either radical surgery, radiotherapy or brachytherapy. We see that the focus of therapy has been whole-gland therapy for disease that might very well be focal. With more widespread screening of healthy asymptomatic men by serum PSA, there has been a paradigm shift in recent years where lower grades and more focal disease are being picked up at an earlier stage. All of these patients do not necessarily require surgery or radical treatment and in fact, if it weren't for PSA screening, most of these patients would have lived the natural course of their lives without the disease manifesting or metastasizing. Thus, just as in breast cancer, a less radical method of treatment, i.e. lumpectomy was required to deal with lower grades of cancer caught in the early stages, a need was felt for non-invasive focal gland therapies for early stage low grade, localised prostate cancer. This is where High Intensity Focussed Ultrasound (HIFU) or Magnetic Resonance Guided Focussed Ultrasound Surgery (MRgFUS) for prostate cancer comes into picture (19).

\section{Technique and Procedure}

MRgFUS is a thermal tissue ablation technique that works non-invasively. In HIFU the high intensity ultrasound waves focussed on a small focal point in the tissue, generate heat by tissue vibration effects to raise the local temperature upto $70-80^{\circ} \mathrm{C}$. This causes thermal coagulation, tissue necrosis and heat shock. The coupled MRI scanner allows accurate targeting of the ultrasound beam by depicting detailed 3D anatomy of the gland. It also helps in monitoring the efficacy of thermal ablation by measuring real time temperatures achieved in the tissue by using real-time MR thermometry.

\section{Selection Criteria}

Currently accepted patient selection criteria for surgery by MRgFUS for prostate cancer are as follows :

1) Age between 55 and 75 years,

2) $\mathrm{PSA}<10$,

3) Gleason Score, $7(3+4)$, 
4) cIIc\& cT2a stage tumors,

5) Maximum of two lesions in the most recent mapping biopsy with each cancer site $<10 \mathrm{~mm}$ in length,

6) Prostate Gland size $<60 \mathrm{~mL}$,

7) No prior TURP,

8) PSAdensity $<0.15$,

9) No extra capsular spread, No calcification in beam path \& tumor $<15 \mathrm{~mm}$.

Patients on medications that could affect PSA values were accepted if those medications were discontinued for at least 3 months prior to the treatment.

\section{Results}

Several reviews and published experience have shown urinary retention \& dysuria, erectile dysfunction and urinary incontinence in a small number. Biopsy proven recurrence rates have also been studied and range from $1-20 \%$ for the entire prostate gland and $1-10 \%$ for the treated lobe.Erectile dysfunction is another complication that has been observed.

A number of urologists have reported negative biopsies of treated lobes in about $80 \%$ of patients.

In a carefully selected cohort of patients with localised prostate cancer of lower Gleason Grade, MRgFUS is a non-invasive technique that provides reasonable cure rates with significantly lower incidence of side effects like incontinence \& erectile dysfunction as compared to conventional methods.

\section{Acoustic Radiation Force Impulse (ARFI) Imaging of Prostate}

ARFI is a novel elasticity imaging technique to detect abnormalitiesinside the prostate. This technique has been extensively used in liver pathologies. ARFI imaging uses high intensity focused acoustic beams to mechanically excite or push tissue and ultrasonic correlation based methods to monitor the tissue displacement response. The results suggest ARFI imaging is a promising new tool for providing image guidance for targeted prostate needle biopsies and focal therapy. This will however need further trials.

\section{Conclusion}

Detection of prostate cancer at an early stage is a boon for men. From DRE andtransrectal ultrasound, we have progressed significantly in the detection and treatment of prostate cancer. Unfortunately the incidence of prostate cancer has significantly increased. It is believed that this is because of early detection of prostate cancer at $\mathrm{T} 1$ stage, unlike previously seen cancers at T2 stage. Whether it is colour Doppler, elastography, HIFU or otherwise, there is significantly better localization of prostate cancerleading tobetter technique of prostate biopsy. A lot of attention has been drawn to the use of contrast enhanced ultrasound for definitely improved delineation of prostate cancer and also for follow up post treatment of these lesions. The addition of Mp MRI and PSMA-PET and the availability of HIFU have been invaluable in both detection and treatment of prostate cancer. Much as has been tried as anterior tumours are often missed on the available imaging techniques. With all these advances, TRUS and PSA will still remain the basic available modalities in spite of their limitations.

\section{References}

1. Taneja SS (2004). Imaging in the diagnosis and management of prostate cancer. Rev Urol 6(3):101-113.

2. Shariat SF, Canto EI, Kattan MW, Slawin KM (2004). Beyond prostate-specific antigen: new serological biomarkers for 
improved diagnosis and management of prostate cancer. Rev Urol 6(2):58-72.

3. Norberg M Egevad L, Holmberg L, Sparen P, Norlen BJ, Busch C (1997). The sextant protocol for ultrasound-guided core biopsies of the prostate underestimates the presence of cancer. Urology 50:562-566.

4. Krucker J, Xu S, Venkatesan A, et al (2011). Clinical utility of real-time fusion guidance for biopsy and ablation. JVIR 22(4):515524.

5. Vourganti S, Rastinehad A, Yerram N, et al(2012).Multiparametric magnetic resonance imaging and ultrasound fusion biopsy detects prostate cancer patients with prior negative TRUS biopsies. J Urol 188(6):2152-2157.

6. Humphrey PA (2012). Histological variants of prostatic carcinoma and their significance. Histopathology 60(1):59-74.

7. Miyagawa T, Tsutsumi M, Matsumura T, et al (2009). Real-time elastography for the diagnosis of prostate cancer: evaluation of elastographic moving images. Japanese $J$ Clin Oncol 39(6):394-398.

8. Cornelis F, Bras YL, Rigou G, Correas JM, Grenier N (2014). Contrast enhanced ultrasound in prostate cancer. In: Image Guided Prostate Cancer Treatments. Bard RL, Futterer J, Sperling D, eds. New York: Springer-Verlag, 47-54.

9. Konig K, Scheipers U, Pesavento A, et al (2005). Initial experiences with real-time elastography-guided biopsies of the prostate. JUrol 174:115-117.

10. Taverna G, Morandi G, Seveso M, et al (2011). Color doppler and microbubble contrast agent ultrasonography do not improve cancer detection rate in transrectal systemic prostate biopsies. BJU Intl 108:1723-1727.
11. Smeenge M, Mischi M, Laguna MP, et al (2013). Contrast-enhanced ultrasonography. In: Current Clinical Urology. Imaging and Focal Therapy of Early Prostate Cancer. Polascik TJ, ed. Totowa, NJ: Humana Press, 155-164.

12. Choi YJ, Kim JK, Kim N, Kim KW, Choi EK, Cho KS (2007). Functional MR imaging of prostate cancer. Radiographics 27:63-75.

13. Hoeks CM, Barentsz JO, Hambrock T, et al (2011). Prostate cancer: multiparametric MR imaging for detection, localisation and staging. Radiology 261:46-66.

14. Weinreb JC, Barentsz JO, Choyke PL, et al (2016). PI-RADS prostate imaging reporting and data system: 2015, version 2 . Eur Urol 69(1):16-40.

15. Roethke M, Anastasiadis AG, Lichy M, et al (2012). MRI-guided prostate biopsy detects clinically significant cancer: analysis of a cohort of 100 patients after previous negative TRUS biopsy. World J Urol 30:213-218.

16. Kallur KG, Ramchandra PG, Rajkumar K, et al (2017). Clinical utility of gallium 68 PSMA PET/CT scan for prostate cancer. Indian J Nucl Med 32(2):110-117.

17. Budaus L, Leyh-Bannurah SR, Salomon G, et al (2016). Initial Experience of (68) GaPSMA PET/CT imaging in high-risk prostate cancer patients prior to radical prostatectomy. Eur Urol 69(3):392-396.

18. Huggins C, Stevens RC Jr, Hodges CV (1941). Studies OnProstate Cancer II.The effects of castration on advanced carcinoma of the prostate gland. ArchSurg 43:209-223.

19. Blana A, Walter B, Rogenhofer S, Wieland WF (2004). High-intensity focused ultrasound for the treatment of localised prostate cancer: 5-yearexperience. Urology 63(2): 297-300. 